\title{
De la cène à la scène : le banquet du Moyen-Âge à Shakespeare
}

Jean-Paul Debax

\section{(2) OpenEdition}

Journals

Édition électronique

URL : http://journals.openedition.org/shakespeare/902

DOI : 10.4000/shakespeare.902

ISSN : 2271-6424

Éditeur

Société Française Shakespeare

Édition imprimée

Date de publication : 1 novembre 2002

Pagination : 31-47

Référence électronique

Jean-Paul Debax, "De la cène à la scène : le banquet du Moyen-Âge à Shakespeare ", Actes des congrès de la Société française Shakespeare [En ligne], 19 | 2002, mis en ligne le 01 novembre 2007, consulté le 03 mai 2019. URL : http://journals.openedition.org/shakespeare/902 ; DOI : 10.4000/ shakespeare.902 


\section{S H A K E S P E A R E \\ \& L E M O Y E N - Â G E}

Société Française Shakespeare

Actes du Congrès de 2001

* * *

Textes réunis et présentés par

Patricia DORVAL

publiés sous la direction de Jean-Marie MAGUIN 


\section{DE LA C ÈNE À LA S C È N E : LE B A N QUET DU MOYEN-ÂGE À S H A K E S PE A R E}

Il n'est pas impossible d'imaginer que l'Angleterre de Shakespeare vivait encore à l'heure médiévale, quoique sans doute moins que les pays catholiques à la même époque. Et quand bien même cela serait, où serait l'infamie ? En tous cas, ce ne sont pas les médiévistes qui y trouveraient à redire ! Encore faudrait-il pouvoir définir le Moyen-Âge, ses bornes et ses caractéristiques pour apprécier sa différence. Il convient plutôt de se méfier de ces distinctions-oppositions suggérées par la formulation «Shakespeare et le Moyen-Âge», qui semble poser d'une part Shakespeare, sous-entendu la Renaissance et son éclat, d'autre part le Moyen-Âge et ses ténèbres, reportant dans le passé de l'Angleterre une espèce de combat des Anciens et des Modernes dont la jeunesse de nous autres Français n'a été que trop bercée, et à le raviver à propos d'un autre pays et d'une autre époque. Laissons donc de côté ces perspectives à proprement parler polémiques ! Je crois personnellement plus aux lentes, mais inévitables, évolutions, aux transitions insensibles, et aux permanences souples qu'aux ruptures brusques et brutales entre des passés oubliés et des avenirs radicalement nouveaux.

L'observation des manifestations de la théâtralité - ou, disons plus simplement des spectacles - en Angleterre au cours des trois siècles qui nous intéressent ici - XIVe, XVe et XVIe - nous convainc aisément que 
l'activité spectaculaire pour une époque donnée est loin d'être monolithique. On peut y déceler des courants parallèles, l'aristocratique et le populaire, le traditionnel et l'humaniste, le chrétien et le folklorique,... qui en fait sont loin de s'ignorer mutuellement et qui, dans une étrange géométrie, se croisent et se superposent parfois, et qui sont repérés à l'aide de noms qu'on a aujourd'hui encore, et malgré de nombreuses et savantes recherches, bien du mal à cerner, et dont l'énumération nous jette dans la même confusion que la liste de Polonius : mystère, miracle, moralité, mumming, disguising, masque, interlude, comedy, tragedy. Toutes ces manifestations de la théâtralité ont des tonalités différentes, des intrigues aussi différentes (quand elles en ont une!), des langues, des styles différents. Mais a-t-on assez réfléchi à la base stable qui fournit un support commun, bien que malléable, à ces diverses expressions artistiques. Je veux parler des contraintes matérielles propres au théâtre, inconnues des genres poétique et narratif, et que nous appelons aujourd'hui la mise en scène. Terme et préoccupation certes étrangers aux différents siècles que nous envisageons ici, mais qui se sont néanmoins impösés dans la pratique dès qu'une réalisation théâtrale a été envisagée.

L'acteur évoluant sur une scène - ou dans un autre lieu - Hall aristocratique par exemple — peut-il jouer de façon prolongée sur un plateau totalement nu, sans mobilier ou autre accessoire qui motive ses gestes ou ses déplacements, ou qui les requalifie en signes théâtraux, facilitant ainsi leur lecture par le spectateur? Le costume, les objets utilisés par les acteurs, le mobilier, le décor y contribuent certainement, encore faudrait-il s'entendre sur les définitions. Je connais peu de travaux dans ce domaine, excepté ceux qui concernent l'arbre théâtral ${ }^{1}$.

Dans la recherche de la permanence, il m'a paru que des éléments moins soumis à la mode que les costumes, et moins artistiquement marqués que les feuillages d'arbre pouvaient mieux servir ma démonstration. Je veux dire le mobilier minimal, la table et le siège, qui semble aller de soi, et par le fait même est rarement l'objet d'une mention particulière dans les textes que nous avons en main aujourd'hui. L'importance de la table et du siège ne devrait susciter aucune surprise chez l'analyste de théâtre, car ce mobilier est la marque fondamentale de la civilisation occidentale. La silhouette humaine, base de la figuration théâtrale, se conçoit difficilement dans nos régions sans l'environnement constitué par ce mobilier minimum qui, dans la réalité extra-théâtrale, était complété par quelques coffres et malles pour le transport des effets personnels et de la vaisselle. 
Ce mobilier est chargé de valeurs symboliques, qu'il va transporter avec lui sur la scène de théâtre. La table évoque le repas pris en commun, en même temps qu'elle constitue un centre spirituel, nous suggère le dictionnaire des symboles de Chevalier et Gheerbrant. Ce sont ces valeurs de participation et de fraternité humaine que nous trouvons incarnées pour la littérature médiévale par la Table Ronde de la légende arthurienne. Le siège, quant à lui, particulièrement s'il est surélevé, est symbole d'autorité, de supériorité. Il s'oppose à la table en tant que symbole d'un privilège, d'une différence. En termes structuraux, on pourrait opposer la verticalité du siège à l'horizontalité de la table, qui concourent ainsi par leur complémentarité à la construction d'un univers signifiant et harmonieux ${ }^{2}$. C'est à l'élément horizontal que constitue la table que je vais consacrer cette étude.

En une démarche à la fois historique et pragmatique, essayons de nous mettre à la place de l'entrepreneur de spectacle, du patron de troupe théâtrale dans le courant du XIVe siècle. Quelle que soit la nature de la pièce qu'il était chargé de monter, mystère, farce ou interlude, cet entrepreneur évoluait dans un cadre urbain où il pouvait le plus facilement du monde trouver tables et sièges dans le monastère le plus proche, l'église ou la cathédrale, ou dans la maison des guildes, ou encore dans l'auberge, dont il utilisait peut-être la cour pour ses représentations. Ces lieux appartiennent à deux domaines conçus comme antagonistes : le domaine du sacré et celui du profane. Le domaine sacré fournit comme modèles la table d'autel et la table du réfectoire monastique ; le domaine du profane la table de banquets et la table d'auberge ${ }^{3}$.

Ces tables provenant de chacun de ces deux univers sont investies de valeurs symboliques opposées, mais dans les deux cas la table est associée à la consommation de nourriture. Bénéfique dans le premier cas : nourriture céleste du sacrifice de la messe, nourriture frugale du réfectoire monastique ; maléfique dans le second : excès de nourriture et de boisson associés à la table de banquet, de même qu'à la table d'auberge, à laquelle s'attachent également des connotations de débauche. L'auberge est «l'école du diable», comme nous le rappelle Langland et l'auteur de Dives et Pauper ${ }^{4}$. Le choix est donc, du moins en théorie, entre banquet divin et banquet infernal.

À côté de la représentation du repas de Pâques, et à la faveur des correspondances typologiques, les pièces religieuses, Passions et Mystères, mettent en scène plusieurs banquets qui sont interprétés comme des analogues de la dernière Cène. Les occasions de proposer des tableaux 
représentant l'ingestion de nourritures ne manquent pas dans les Évangiles : noces de Cana, manne dans le désert, multiplication des pains, repas chez Simon, chez le publicain généreux, à Emmaüs. Les facteurs de mystères ne se sont pas fait faute de céder à la tentation que représentaient ces occasions de mises en scène spectaculaires. Ainsi les instructions contenues dans le manuscrit de la Passion de Mons nous renseignent sur le détail des mets fournis aux acteurs pour chaque journée. Pour les noces de Cana : épaule d'agneau, poitrine de veau, pâté de poulet, des gâteaux et des poissons pour le miracle de la multiplication des pains (Mat. 14.15-21). D'autres nourritures sont mentionnées pour le banquet d'Hérode après la décollation de Saint Jean Baptiste, et pour le repas avec Simon le Lépreux (Mat. 26.6 ; Marc. 14.3 ; et (?) Jean. 12.1). Toujours à Mons, mention est faite du mobilier : pour la scène de la célébration de la Pâques par Jésus et les apôtres, un buffet est transporté sur le théâtre par une voiture, deux tapisseries sont empruntées, l'une à la maison d'un seigneur, l'autre à l'église Saint Nicolas. Il n'est pas question de tables, pourtant indispensables en cette occasion, probablement parce qu'elles devaient être disponibles et donc ne coûtaient rien, ni en frais de transport ni de location.

Particulièrement intéressante à ce propos est la disposition des lieux sur le plan de la place où se joue la Passion de Lucerne. Une table reste en place pendant les deux journées des représentations, qui était utilisée successivement, le premier jour pour le repas chez Simon le Lépreux (épisode où Marie Madeleine lave et parfume les pieds du Christ) et chez Zachée (riche et généreux Publicain qui invite Jésus lors de son passage à Jéricho, alors que rien n'est dit dans l'évangile sur l'organisation d'un repas à cette occasion (Luc, 19.1-10). Pour le deuxième jour, la table servira pour le repas chez Lazare (compris sans doute comme différent de celui qui a lieu chez Simon, bien que les évangiles ne soient pas clairs à ce sujet), et bien entendu pour le dernière Cène. Une autre table servira au repas à Emmaüs après la Résurrection (Luc, 24.13-35).

Le repas de la dernière Pâque est le banquet sacré par excellence. Il est représenté dans la grande majorité des Passions et, en ce qui nous concerne, dans tous les cycles anglais de Mystères sauf un, celui de Wakefield. Dans la pièce de $\mathrm{N}$ Town, la didascalie indique que les disciples sont découverts en scène «sytting at the table» (Passion II.669). La pièce $\mathrm{XV}$ de Chester donne des détails sur la préparation du dernier repas dans la maison de celui que les évangiles nomment l'homme à la cruche. Cet hôte déclare : 
Loe, here a parlour ready dight

With paved floors and windows bright

Pour ne pas paraître insolite cette description implique forcément une mise en scène minutieuse, et le vers suivant :

Make things ready, as you think right

laisse supposer la préparation d'un vrai repas, au vu des menus détaillés décrits dans les instructions pour la Passion de Mons déjà citées, auxquelles on peut ajouter des précisons données pour la Cène de Lucerne : «le sauveur découpe l'agneau pascal», dont les convives ne laisseront «que les os», et qu'ils accompagneront de «laitue sauvage et de pain azyme».

Dans les cycles anglais, comme dans les Passions continentales, la Cène n'est pas le seul repas pris en public, ou évoqué. À Chester, le Seigneur ressuscité, mais qui n'a pas été encore reconnu par ses disciples, est invité par Luc et Caïphe à se rendre au «Castellum» (il s'agit d'Emmaüs) pour y prendre un repas, où Luc lui dit :

Sitt down, Sir, here, I you pray

And tak a morsell, if you may

(XIX.113-4)

La didascalie indique alors : «Tunc frangit panem et ait»,

Jesus. Eates on, men, and doe gladly

In the name of Good Almighty.

(XIX.121-2)

N'y avait-il que du pain au menu ? Le pain est évidemment indispensable pour rappeler la consécration instituée lors de la Cène eucharistique, mais tout porte à croire qu'un repas complet l'accompagnait. D'ailleurs, tandis que Cleopas rapporte l'événement aux autres apôtres, parmi lesquels Pierre, Jésus apparaît à nouveau, et un second repas est organisé par Pierre, qui comprend : 
Rosted fishe and hony, in feere,

Therof we have good wonne* * (store)

(XIX.195-6)

Tunc comedet Jesus, et dabit discipulis

(XIX.200)

Le même épisode est relaté dans $\mathrm{N}$ Town; le même pain partagé (N Town, 38.206, 216, 284-8). Le repas à Emmaüs de la pièce de York présente l'intérêt de mentionner la table sur laquelle est déposé le pain que le Christ va rompre et distribuer :

Now bless I this bread that brought is on the board

(voir 42.63-84, pour la description d'un deuxième repas composé de miel et de poisson, et Wakefield 29.255-6, 279-90, où plus explicitement encore, Cleophas déclare : «lo, here a board and cloth is laid / And bread thereon freshly arrayed», 285-6). Tous ces exemples mettent en lumière que dans les pièces de Corpus Christi la mise en scène du repas est liée au mystère de Jésus et à l'efficacité de la Rédemption et qu'à cette occasion la présence d'une table sur scène accompagne toujours cette solennité.

Plus surprenant est cet autre repas, celui des bergers de Coventry, de Wakefield et de Chester, attendant la naissance du Sauveur. Il ne constitue pas ouvertement une célébration, il n'est pas non plus cautionné par un texte évangélique, et semble donc devoir son existence à une pure dynamique dramatique, ou peut-être à une cohérence homilétique. Après un dialogue introductif sur les rigueurs du temps, sur la dureté de la condition populaire au ton désenchanté, dicté sans doute par la tradition de la Pastorale dont nous ne savons que peu de choses, soudain l'un des bergers de Coventry annonce son désir de se restaurer :

Here awhyle lett us rest

And repast owreself of the best

(Shearmen \& T. 238-9) 
La didascalie ajoute : «There the scheppardis drawys furth there meyte and doth eyte and drynk». Il y a donc bien consommation sur scène. S'agit-il du petit en-cas matinal que prend le paysan avant d'attaquer le travail ? Ce n'est pas impossible, mais il y a peut-être aussi une autre raison.

Remarquons en outre qu'un berger de Wakefield hèle, contre toute vraisemblance réaliste, un de ses compagnons par «are ye in this town?». Si ses paroles ne conviennent pas à sa situation de berger gardant ses troupeaux sur les collines de Bethléem, en revanche, elles sont en accord avec la situation de l'acteur engagé par l'entrepreneur d'un festival urbain (cf. aussi Chester, VI.283). Dans Wakefield, la conversation des bergers commence par un monologue du vendeur d'aromates, ou «mire», constitué par une énumération des noms d'herbes médicinales, rappelant le catalogue de l' «espicier», fréquent dans les Passions continentales dans la scène où les Maries s'avancent vers le tombeau du Christ après la résurrection, ou scène du Quem Quaeritis (Chester, VII.17-92). La présence de ce catalogue constitue une preuve de contamination, d'envahissement du présent épisode par des matériaux traditionnels et étrangers, comme plus loin la scène de lutte provoquée par le jeune berger Trowle, et comme l'est aussi la joute verbale (flouting) à laquelle les bergers se livrent en permanence; de même encore l'exécution d'une chanson dans la Seconda Pastorum de Wakefield (Trowle en déplore l'absence dans Chester, VII.216).

La pièce de Chester nous fournit le menu :

$2^{\circ}$ Pastor. Here is bread this day was baken, Onyons, garlik, and lyckes, Butter that bought was in Blacon, And greene cheese that will grease your cheekes.

$3^{\circ}$ Pastor. And here ale of Halton I have, And what meat I had to my hyre ; A pudding maye no man deprave, And a Ianock* of Lancashyre. *oat-cake (?)

Lo ! here a sheepes head souced in ale, And a groyne to lay on the grene, And soure mylke my wife had on sale : A noble supper as well is seene.

$1^{\circ}$ Pastor. And as it is well sene, ya shall see And what somewhat I have in my sacke : 
A piggs foote I have here, pardye !

And a panch cloute in my packe.

A womb clout, fellowes, now have I,

A lyver as it is no lack,

A chitterling boyled shall be.

This burden I beare on my backe.

Nowe will I cast of my cloake, And pull out parte of my livery, Pull out that I have in my poke, And a piggs foote from pudding purie.

$3^{\circ}$ Pastor. Abyde, fellowes, and ye shall se here, This whot meat, we shall it hend, Gambons, and other good meat in feare, A pudding with a prick in the end.

$1^{\circ}$ Pastor. And that is in my sachell to shake out, To shepheardes am I not ashamed :

This ox tonge, pared round about, For your tooth it shall be attamed. (Tunc comedent)

(VII.113-44)

Le même souci du détail du menu se retrouve dans Wakefield 12, quand, avec un effet parodique (ou profondément révélateur d'un sens caché ?), le premier berger s'écrie : «is this a church rite ?» (211-49).

Nous ne somme pas au bout de nos surprises. Dans la même pièce, le premier berger s'exclame, regardant le sol : «what a turd» (193), et fait la suggestion suivante :

I'd rather eat.

What ! Drink without meat?

A meal I entreat,

And set up a board,

Then may we so dine our bellies to fill

(194-8)

Eh, oui ! Le berger réclame une table pour étaler ses victuailles et organiser un festin. Une table sur les collines de Bethléem ! Il y a sans doute une profonde ironie dans cette invitation incongrue dans un cadre 
rural (comme l'est la référence à la ville, déjà citée), mais qui est loin d'être dénuée d'objet ni de signification. En effet, le comportement de ces bergers dans les trois Cycles utilisés (disputes, joutes verbales, chants collectifs et lutte) est celui des rustres se retrouvant dans l'auberge locale un jour de fête, lieu où, bien entendu, les tables sont de rigueur. Il ne fait pas de doute qu'à ce moment-là de la pièce concernée, des tables étaient avancées et une sorte de salle d'auberge évoquée sur scène. Cette auberge n'est pas neutre ou simplement folklorique. Elle sera interprétée d'abord en fonction de l'image négative, école de Satan, colportée par les textes homilétiques, et c'est à un anti-banquet eucharistique que se livrent les bergers.

La qualité parodique de ces scènes ne fait pas de doute, si on considère la succession des deux pièces de bergers de Wakefield. La deuxième, la secunda pastorum, est plus connue : c'est celle où Mak cache dans un berceau l'agneau qu'il vient de voler aux autres bergers, proposant ainsi l'image d'une anti-Nativité. Mais ni l'une ni l'autre de ces deux parodies n'est proprement diabolique. Elles constituent plutôt la version familière, ou populaire de l'original sacré ou édifiant, peut-être héritée de la tradition goliardique. Elles sont comme un repoussoir, une image imparfaite qui fait ressortir, par contraste, l'image de l'idéal souhaité, mais toujours inaccessible pour le commun des mortels, et concourent, tout compte fait, à la célébration de l'excellence du plan divin.

Les scènes d'auberge proprement dites sont loin d'être inconnues dans le théâtre moral du Moyen-Âge depuis son apparition au XIIIe siècle dans le Nord de la France. C'est dans un tel décor que se déroulent les trois quarts du texte de Courtois d'Arras, pièce traitant du thème du Fils Prodigue, et privilégiant l'épisode de vie dans la débauche, qui est représentée par une longue scène de cabaret / bordel. Quoiqu'insérée dans une intrigue morale, cette scène a cependant des vertus comiques certaines et un charme propre.

La scène d'auberge dans Mary Magdalene de Digby est plus ouvertement moralisante. Elle est précédée d'un monologue du genre «bateleur» prononcé par l'aubergiste qui vante ses vins, comme l'«espicier» du Quem Quaeritis vantait ses aromates. C'est une scène de séduction et de dissipation, où Marie cède aux avances du galant Curiosity. Des formules comme «go we ner» et «bring us» $(482,484)$, utilisées par Luxuria indiquent que ces deux personnages s'étaient assis à une table de l'auberge. L'auberge est également le lieu des plaisirs et de la damnation dans Nature de Medwall. Sensuality relate la rencontre dans 
une taverne entre le héros «Man» et deux demoiselles de petite vertu, Kate et Margery, dont il tombe amoureux, consacrant ainsi la victoire de ses mauvais penchants $\left(1^{\circ}\right.$ partie, 1038). Par un effet d'emboîtement homothétique, on peut imaginer que le récit qu'en fait Sensuality, à Orgueil (Pride) et à Amour Mondain (Worldly Affection), se déroule aussi dans une taverne, lieu qui sied parfaitement à leur qualité de mécréants et de joyeux lurons. Il faut mentionner également un banquet maléfique déguisé : celui de la célébration de la Pâque, disqualifié car présidé par Hérode (N Town, 31 ; cf. Archipropheta de N. Grimald) et qui constitue le pendant inversé du repas eucharistique.

Dans tous les cas le lien typologique est manifesté par la permanence de l'image, la présence de l'accessoire commun à tous ces épisodes, la table, qui permet de construire un espace spécifique, aisément repérable, qui met le spectateur dans une situation d'attente, et balise la réception du public du théâtre religieux, parallèlement à d'autres dispositifs; comme celui du tribunal, qu'on trouve dans le procès de Marie et de Joseph, le Procès de Paradis, les procès du Christ et le Jugement Dernier.

$* * *$

Entre le théâtre religieux des Cycles et les pièces du XVIe siècle, il existe une véritable solution de continuité, provoquée en grande partie, on imagine, par des destructions massives de textes de pièces qui restaient en manuscrits dans les malles des troupes d'acteurs. Une exception cependant, parmi les pièces dites «bibliques», en général de tonalité protestante, des années 1550-60, The Life and Repentence of Mary Magdalene, de L. Wager, comporte un repas pris en scène (appelé «repast», 1487 et 1514), le repas chez Simon, mais n'apporte aucun progrès par rapport à la pièce de Digby.

Nous avons à prendre en considération maintenant un nouveau terme : le mot «banquet», qui jusque-là, n'avait pas paru dans le théâtre anglais. Et c'est bien normal, puis qu'il n'existait pas dans l'usage courant. Les grandes ripailles royales étaient désignées par les termes «diner», «fest'», «repast», «mirthe», ou encore «mete». «Banquet'» est un emprunt tardif (OED donne 1483, Caxton), de l'italien «banchetto» diminutif de «banco». Il est introduit en anglais en même temps que se répandent de nouveaux mets et de nouvelles modes alimentaires. Le premier emploi au théâtre est, à ma connaissance, dans Goodly Queen Hester (1527), pour désigner le festin qui est aussi appelé «repast» dans le dialogue (895). Pour 
résumer, disons que le changement a pour origine l'introduction du sucre. Sous l'influence des pays orientaux, des populations arabes par l'intermédiaire de l'Espagne et du Portugal, le sucre perd peu à peu sa qualité exclusivement médicinale pour devenir une friandise, de grand luxe dans un premier temps, à cause de son prix et de son image exotique, qui se démocratise à l'époque élisabéthaine jusqu'à se répandre dans la classe bourgeoise riche. Le sucre reste pendant toute la période synonyme de sophistication et de luxe, de même que les vins (qui s'opposent à la bière autochtone), et les compositions à base de vin et de sucre (clairé et hypocras) ${ }^{5}$.

C'est cette idée de luxe qu'exprime le terme «banquet». Comme la plupart des termes d'emprunt il projette une lumière valorisante sur l'objet désigné, mais en même temps ce terme a du mal à trouver sa place dans le lexique anglais et est entouré assez longtemps par un certain flou référentiel. Pour ma part, je distinguerai trois acceptions :

1. Il est pris comme équivalent de «feast, meal, meat», et le «banqueting hall» ou «house» est une nouvelle dénomination de ce qu'on appelle encore dans les collèges universitaires le «dining hall», c'est-à-dire la grande salle où se tenaient les repas de cérémonie ainsi que les fêtes, danses, disguisings et masques.

2. Il désigne un repas séparé constitué de sucreries, de compotes, de fruits confits et autres pâtisseries à base de sucre, pris en général dans le courant de l'après-midi et servi dans un lieu séparé appelé «banqueting room» ou «banqueting house», ou encore «pavilion» s'il se situe dans le parc.

3. Il désigne la partie terminale du repas, qui jusqu'au début du XVIe siècle, n'avait ni existence systématique, ni nom spécifique, et qui est constituée de sucreries diverses, et est servie à la suite du repas. Le terme «dessert» n'apparaît en anglais qu'en $1600(O E D)$ et est absent de l'œuvre de Shakespeare. C'est aussi un terme d'emprunt : mot français qui succède au médiéval «voidée» qui signifie «desserte», cette partie du repas étant consommée après que les tables ont été débarrassées des plats de viandes.

Le terme valorisant de «banquet» envahit donc le théâtre de l'époque Tudor, pour désigner toute consommation formelle de nourriture sur scène et nécessitant l'emploi de tables. Les exemples sont nombreux, et nous n'en examinerons ici que quelques échantillons. 
On se souvient que, dans As You Like It, un banquet est préparé dans la forêt sur ordre du Duc Senior (II.5.58). Orlando parvient à l'endroit du banquet avec le vieil Adam mourant de faim. Il est poliment invité à partager les victuailles par le vieux Duc, et il s'étonne aussitôt de la politesse avec laquelle il est reçu :

Speak you so gently? Pardon me I pray you

I thought that all things had been savage here

(II.7.106-7)

Le banquet est donc l'occasion de témoigner de la civilité et du raffinement. Le banquet est la manifestation spectaculaire de la supériorité morale qui permettra au Vieux Duc de triompher à la fin de la pièce sur son frère l'usurpateur. Nous remarquons également que cette scène est entourée de discours prônant la supériorité de la civilisation urbaine, et le festin est associé à la représentation théâtrale dans le fameux monologue de Jaques sur le théâtre qui suit immédiatement la scène du banquet.

Dans The Taming of the Shrew, comme d'ailleurs dans The Taming of $\underline{A}$ Shrew, Sly est persuadé qu'il est devenu un seigneur par la vue d'un banquet qui, est-il précisé, est constitué par une table devant laquelle on le fera asseoir. Mieux, il se croira au ciel (44), preuve de la persistance de l'association religieuse, souvenir de la table eucharistique. La didascalie précise qu'il s'agit bien d'une table, sur laquelle le banquet est disposé. Comme à Mons, nous connaissons le menu du banquet de $\underline{A}$ Shrew : une épaule de mouton, du vinaigre pour faire rugir le Diable et, bien entendu, du vin $(87-8,92,132)$. Du sack et des sucreries sont mentionnées dans The Shrew (2 Induc., 2-3). Ici encore, le repas est comparé à une représentation théâtrale.

Dans Pericles un banquet de célébration fait suite à la victoire de Périclès dans le tournoi (II.3), et est lui-même suivi d'un bal (III.2). Ces deux réjouissances sont présentes dans Romeo and Juliet, quoique dans l'ordre inverse (I.5); mais le banquet écourté laisse présager d'une fin tragique (voir aussi Much Ado, le masque, II.1, est suivi d'un banquet en coulisses, fin de II.1).

Cette conjonction du banquet et de la fête (masque, danse), est sans doute due à la familiarité croissante des acteurs avec les palais et la cour, et à l'influence donc du déroulement de la fête aristocratique, qui reflète le raffinement des mœurs. En ce qui concerne le banquet proprement dit, j'en distinguerai trois variétés : 
1. le banquet de célébration, ou de solennisation d'un événement. La première occurrence chronologique nous en fournit un exemple. Dans Goodly Queen Hester, Esther invite son époux à un banquet pour lui faire la demande formelle de protéger ses coreligionnaires menacés par le ministre Aman. C'est aussi le but du banquet organisé à bord de la galère de Pompée, dans Antony and Cleopatra, pour sceller l'amitié entre Octave, Antoine et Pompée lui-même. Il se transforme rapidement en beuverie de bas étage qui évoque plus la scène de cabaret que le festin royal. Une conclusion théâtrale nous est suggérée par l'appel à se donner la main, tels des acteurs qui saluent après le dernier rideau $(107,125)$; mais la danse qui termine ces agapes est plutôt un anti-masque grotesque, comme l'indiquent les termes de «bachanals» et de «antick» $(103,124)$.

2- Le deuxième type est le banquet d'amour, où c'est Éros qui se manifeste sous la forme en général associée à l'Agapé ${ }^{6}$. L'inspiration en vient peut-être du Cantique des Cantiques, qui décrit l'amour comme consommation de vin et de fruits (Cantique, 2.3-4). Shakespeare en donne une version ironique dans le banquet avorté de Antony and Cleopatra, qui devait célébrer la rencontre des amants. On peut imaginer que les préparatifs se poursuivent au vu du spectateur tandis que Charmian demande au Voyant de lui prédire l'avenir, mais l'ombre de Fulvia vient aussitôt gâcher cette perspective de réjouissances. Les véritables banquets d'amour n'apparaîtront au théâtre qu'après Shakespeare. Le plus caractérisé est celui de The Insatiate Countess de Marston (1613), où le mot «amour» peut être pris dans son sens le plus plein et le plus complet, le banquet dégénérant (ou culminant !) en véritable partie fine entre Isabella et sa nouvelle conquête, Gniaca. Dans le même esprit, le banquet de la Tragedy of Messalina, plutôt connue, elle, comme «insatiate empress». D'autres pièces, en particulier celles de Fletcher, utilisent cette même scène pour des épisodes de séduction, tout en allant moins loin dans le réalisme !

3- Le troisième type est ce que j'appellerai le banquet diabolique, qu'il soit à proprement parler d'origine infernale ou plus vaguement néfaste. Particulièrement intéressant est le banquet invisible de Macbeth dont nous ne voyons que l'envers, pour montrer le dérèglement général entraîné par le projet criminel de Macbeth et de son épouse (I.7). Rite de célébration occulté, refusé au spectateur qui ne verra que le va-et-vient des serviteurs, les angoisses et les pensées meurtrières du couple. Le deuxième banquet de la pièce est plus célèbre. Il est censé être organisé pour solenniser l'accession de Macbeth au trône d'Écosse. Certes, Shakespeare 
a trouvé le nom de Banquo dans Holinshed, et il ne dérive donc pas du banquet fantomatique auquel ce dernier participe post mortem. C'est peutêtre inversement ce nom qui a suggéré au dramaturge de figurer sous forme de banquet les remords suscités chez Macbeth par le meurtre de ce compagnon innocent, puisque Macbeth avait précédemment déclaré son amitié pour Banquo en ces termes, clairement prophétiques : «He is a banquet to me» (I.4.54).

Un dernier avatar du banquet s'inscrit dans cette suite de réalisations scéniques, le banquet de punition, dont la forme la plus spectaculaire est le «banquet sanglant», ou banquet cannibale. Je ne m'étendrai pas sur le sujet, car je n'ai que fort peu à ajouter à la récente et excellente thèse de L. Niayesh ${ }^{7}$. Nous avons dans ce cas des banquets au cours desquels est «consommée», au sens littéral du terme, la vengeance, sous forme du corps d'un ennemi ou d'une victime. On peut évoquer d'abord deux cas transitionnels. D'abord, dans la Spanish Tragedy, le banquet de l'acte I est certes un banquet de célébration, donné en l'honneur des ambassadeurs portugais. Il est suivi d'un masque organisé par Hieronimo, en apparence pour sceller la réconciliation entre Espagnols et Portugais, mais on ne peut ignorer qu'il porte en germe la «tragédie» finale, conçue par le même Hieronimo, qui apportera un dénouement sanglant à la pièce. Les banquets de Timon of Athens sont peut-être parmi les plus intimement intégrés à une action dramatique, qui n'est d'ailleurs que très marginalement tragique. Le premier banquet est celui de la duperie de Timon par les flatteurs, au cours duquel il met le clairvoyant mais cynique Apemantus à l'écart, en le faisant manger tout seul à une table séparée. Le deuxième banquet fournit l'image de la punition de ces mêmes flatteurs par Timon, opérée par la dérision, lorsqu'il leur sert des plats d'eau chaude, qu'il finit par leur lancer à la tête. Nous avons peut-être atteint là le dangereux point où le banquet se vide de contenu, n'étant plus que pur spectacle, et risque de se nier en tant que forme d'expression. On peut rapprocher le banquet de Timon du «méta-banquet» de $A$ Warning for Fair Women, qui se présente comme une scène muette, dumb show, jouée par des. personnages allégoriques, qui interrompt l'action, et fait l'objet d'un commentaire moral sur l'action plus réaliste de l'intrigue, énoncé par le personnage de la tragédie.

On ne peut pas prétendre que le premier banquet de Titus Andronicus soit un banquet euphorique. Titus y paraît avec sa main coupée, après qu'un messager lui a apporté deux têtes, celles de ses deux fils, et une main, sa propre main, que l'empereur a refusé d'accepter pour l'humilier. 
De plus, ce banquet est marqué par l'incident de la mouche tuée par Marcus, frère de Titus, et qui préfigure les meurtres à venir au cours du banquet final. De la même manière, le banquet est aussi un masque et presque un dumb show au cours duquel la vengeance va se réaliser.

Nous sombrons alors dans le banquet sanglant, à la Sénèque, avec le deuxième banquet de Titus: six morts, plus le sang des deux fils de Tamora servi sous forme d'un petit pâté. Nombreux sont les exemples. Pour en rester aux pièces les plus connues en dehors de Shakespeare, citons Antonio's Revenge, où les membres de Julio sont servis en fricassée à son père Piero Forza, le cruel tyran de Venise. Ce banquet faisant luimême partie d'un masque. Pour conclure, nous mentionnerons le banquet de la pièce de 1639, qui a pour titre The Bloodie Banquet (1639), où la reine du tyran est forcée de manger sur une petite table à part, comme Apemantus de Timon of Athens, les membres de celui qui est accusé à tort d'être son amant.

$$
* * *
$$

Il est impossible de faire une recension plus détaillée des banquets montés sur scène pendant les périodes Tudor et Stuart, tant la matière est abondante. On est frappé par le nombre de pièces, au moins 80 citées par le Dictionary of Stage Directions de A. Dessen. La fréquence du dispositif scénique consistant à consommer publiquement des nourritures sur des tables ne me paraît pas être le fruit du hasard ou d'une mode. Il paraît évident que, malgré la solution de continuité entre les pièces du MoyenÂge finissant et celles de l'époque Tudor, il y a permanence du dispositif en question, et non pas re-création au cours de la deuxième période.

Cette permanence semble être due à l'utilisation de l'accessoire table, qui a une double fonction, celle de symboliser la consommation de nourritures, et de favoriser l'échange verbal. La fréquentation de l'auberge, comme le rite chrétien comprennent une consommation conviviale de nourritures en même temps qu'une utilisation de la parole. Cette seconde activité est directement transposable au théâtre. Quant à la première, elle semble au contraire incompatible avec le caractère illusoire des créations dramatiques. Les fonctions ou situations humaines fondamentales, au nombre desquelles je compterai l'alimentation, la relation sexuelle et la mort, constituent comme des tabous que la pratique théâtrale est toujours tentée de violer. La mort, on peut en jouer (comme Falstaff sur le champ de bataille de Shrewsbury), ou la donner à voir 
«presque comme vraie», dans un deuxième degré d'illusion dramatique (comme dans le final de la Spanish Tragedy). La représentation de la relation sexuelle paraît de plus en plus au centre des préoccupations des metteurs en scène contemporains. En ce qui concerne la nourriture, le mythe chrétien de l'Incarnation, prolongé par le rite de l'Eucharistie, lui a donné dès les débuts du théâtre occidental une caution et une justification.

Cette consommation de nourriture pratiquée en scène, fascine toujours les spectateurs car, en dehors de tout dogme particulier, elle représente la force vitale, et est donc perçue comme propre à solenniser pour l'œil et pour l'imagination les sommets dramatiques d'un spectacle.

Des collines de Bethléem où, au mépris de toute vraisemblance, les bergers étalent leurs victuailles sur des tables, jusqu'aux horreurs baroques des banquets sanglants des drames de la vengeance, le motif que nous venons d'étudier répond au même besoin de spectaculaire et au même sens du sacré.

Jean-Paul DEBAX

Université Toulouse-le-Mirail

\section{N O T E S}

'Peu de travaux sont à signaler dans le domaine des accessoires de théâtre. Une excellente et récente étude de M.-H. Besnault, «Of Trees Theatrical, symbolic and allegorical in Tudor Drama», Tudor Theatre, Vol. 5, «Allegory in the theatre», Bern, etc., Peter Lang, Coll. Theta, 2000, p. 207-34. Nous avons tous une grande dette vis à vis de la «pioneering study» de Werner Habicht, «The Tree Properties and Tree Scenes in Elizabethan Theater», Renaissance Drama, IV, 1971, p. 61-92. Sans oublier George F. Reynolds, «Some Principles of Elizabethan Staging», MP, Part I.2, 1905, p. 581-614 ; Part II.3, 1905, p. 69-97. Cette étude doit beaucoup à ces prédécesseurs.

${ }^{2} \mathrm{La}$ verticalité renvoie évidemment à l'échelle, ou chaîne, des êtres qui, d'après St Thomas, classe la création depuis le Trône divin jusqu'au centre infernal de la terre. Cette dimension vient entrer en conflit, et aussi compléter, l'horizontalité qui symbolise les échanges entre les humains, dans un ordre qui ne 
représente pas la hiérarchie des créatures, mais les relations d'égalité, ou de convivialité. Voir E. Konigson, L'espace théâtral médiéval, Éditions du C.N.R.S., Paris, 1975, p. 209 ; et Arthur O. Lovejoy, The Great Chain of Being, Harper \& Row, New York, 1960 (1936), Ch. III.

${ }^{3}$ On peut considérer l'autel comme le lieu de rencontre des deux axes déjà mentionnés; là où le dieu transcendant se fait homme, et où est prononcé son engagement de rester homme parmi les hommes sous forme matérielle.

${ }^{4}$ Dives and Pauper, Priscilla Heath Barnum (ed), O.U.P., 1976, EETS, OS, 275 ; W. Langland, Piers the Plowman, W.W. Skeat (ed)., O.U.P., 1953 (1869). L'expression se trouve également dans le pénitentiel Ignorancia Sacerdotum, Phyllis Hodgson (ed)., RES, 1948, XXIV, p. 1-11 : «The Taverne may wel be called the devels scole and his chapel where his scolers rede and sing and where he doth his miracles...».

${ }^{5}$ Voir à ce sujet $\mathrm{C}$. Anne Wilson, «The Origin of Banquetting Stuffe», in A.Wilson (ed), Banquetting Stuffe, Edinburgh U.P., 1991, et C.D. Meads, «Banquet Scenes in English Drama, 1582-1642», thèse non publiée, Université de Birmingham, 1988. On consultera avec profit l' article «banquet» dans A.C. Dessen et L. Thomson, A Dictionary of Stage Directions in English Drama, 1580-1642, Cambridge U.P., 1999, p. 19-20.

${ }^{6}$ D.K. Anderson, «The Banquet of Love in English Drama, 1595-1642», $J E G P, 63,1964$, p. $422-32$.

${ }^{7}$ Ladan Niayesh, «Aux frontières de l'humain», thèse non publiée, Université de Montpellier, Janvier 2000. 